\title{
Application of minocycline in patients with Helicobacter pylori infection allergic to penicillin
}

\author{
Lingyun ZHANG ${ }^{1}$, Yu LAN ${ }^{1 *}$ (D), Qi WANG ${ }^{1}$, Xiaobei SI ${ }^{1}$, Yuexia ZHANG ${ }^{1}$
}

\begin{abstract}
To observe the eradication rate, safety and compliance of bismuth quadruple therapies based on minocycline in the newly diagnosed patients with Helicobacter pylori $(H p)$ infection. We selected 150 penicillin allergic patients for the initial treatment of Hp infection treated in Beijing Jishuitan hospital from January 2018 to February 2019. Subsequently, the patients were randomly selected into the minocycline and metronidazole-containing group (RMMB) and minocycline and levofloxacin-containing group (RMLB). The total course of treatment is 14 days. Follow-up clinical manifestations 3 to 5 days after treatment. The ${ }^{13} \mathrm{C}$ urea breath test was performed 4 to 12 weeks to confirm the eradication of $H p$. There were no significant differences in baseline data between the two groups ( $p>0.05)$. According to ITT analysis, the eradication rates of RMMB group and RMLB group were $80.3 \%(61 / 76)$ and $89.2 \%(66 / 74)$, respectively $(\chi 2=2.301, \mathrm{p}=0.129)$. According to $\mathrm{PP}$ analysis, the eradication rates of the RMMB group and the RMLB group were $83.6 \%$ (61/73) and $90.4 \%(66 / 73)$, respectively $(\chi 2=1.513, p=0.21)$. The overall incidences of adverse events were $47.4 \%$ and $38.8 \%$ in the RMMB and RMLB groups, respectively ( $\mathrm{p}=0.924)$. In patients with penicillin allergy, the eradication rate of minocycline-based bismuth quadruple therapies is ideal and the side effects are few. It is recommended for the eradication treatment of $H p$-infected patients with penicillin allergy.
\end{abstract}

Keywords: Helicobacter pylori; minocycline; bismuth quadruple protocol; penicillin allergy; levofloxacin; metronidazole.

Practical Application: For the eradication treatment of $\mathrm{Hp}$-infected patients with penicillin allergy.

\section{Introduction}

Helicobacter pylori $(H p)$ infection is a global disease. It has attracted attention due to peptic ulcer, gastric cancer, gastric mucosa-associated lymphoid tissue (MALT) lymphoma and other intra- and extra-gastric diseases (The Chinese Society of Digestive Diseases, 2017). However, in recent years, due to the increased resistance rate of $H p$ to various antibiotics, eradication therapy has become more and more difficult. Especially for patients who are allergic to penicillin, there is a lack of ideal treatment options. In many guidelines, tetracycline is recommended as a replacement for amoxicillin for eradication therapy in patients with allergies to penicillin (The Chinese Society of Digestive Diseases, 2017). However, tetracycline is difficult to obtain in China due to adverse reactions. Minocycline is a semi-synthetic tetracycline with fewer side effects. In vitro susceptibility studies found that $H p$ is more sensitive to minocycline, similar to tetracycline (Bai et al., 2015). Our previous study showed that the bismuth quadruple therapies with minocycline/amoxicillin has a good eradication effect on patients with $H p$ retreatment (Zhang et al., 2015a, 2017). Therefore, this study intends to use minocycline instead of tetracycline to observe the eradication effect of minocycline combined with metronidazole and fluoroquinolone on patients allergic to penicillin.

\section{Object and method}

\subsection{Research object}

We selected $H p$-infected patients who were admitted to Beijing Jishuitan Hospital from January 2018 to February 2019. Inclusion criteria: $(1){ }^{13} \mathrm{C}$ urea breath test $\left({ }^{13} \mathrm{C}\right.$-UBT) test, gastric mucosal tissue rapid urease test or pathological section staining positive patients; (2) had not received $H p$ eradication therapy; (3) age 18-75 years old; (4) penicillin allergy; (5) fully informed and agreed to participate in the study. Exclusion criteria: (1) taking drugs that may affect the results of the study 4 weeks before enrollment, such as proton pump inhibitors (PPIs), bismuth or antibiotics; (2) patients with malignant tumors; (3) history of upper gastrointestinal surgery patients; (4) patients with systemic diseases such as severe liver and kidney damage; (5) those who are allergic to the drugs used in this study; (6) pregnant and lactating women; (7) long-term use of glucocorticoids or nonsteroidal anti-inflammatory medicine patient.

\subsection{Grouping and treatment options}

Patients were randomized according to a random number table. Specific drug regimen of minocycline combined with metronidazole group (RMMB): rabeprazole enteric-coated 
tablets (Essence Chinese Pharmaceuticals, 10mg, twice daily) + minocycline (Wyeth-Hundred Palace, $0.1 \mathrm{~g}$, twice daily) + metronidazole (Shanxibao Pharmaceutical, $0.4 \mathrm{~g}$, three times a day) + bismuth potassium citrate (Lizhu Pharmaceutical Co., Ltd., 220mg, twice daily); total course of treatment is 14 days. The specific protocol of minocycline combined with levofloxacin group is to replace metronidazole in the above scheme with levofloxacin (First Sankyo Pharmaceutical (Beijing) Co., Ltd., 0.4 g once daily), and other therapeutic drugs with minocycline is the same as RMLB group.

\subsection{Efficacy and safety assessment}

The adverse reactions and compliance of the patients were followed up 3 to 7 days after the end of treatment; We reviewed the patient ${ }^{13} \mathrm{C}$-UBT in $4-12$ weeks. If it is negative, it is judged that $H p$ is eradicated. $H p$ eradication rates were calculated using intention to treat analysis (ITT) and follow-up protocol analysis (PP), respectively. Compliance is calculated as the percentage of the number of tablets actually taken by the patient in the number of tablets planned to be taken. Less than $80 \%$ compliance is considered poor compliance and is not included in PP analysis (Lesaffre and de Klerk, 2000)

\subsection{Statistical analysis}

The statistical analysis of this study used SPSS (version 19, SPSS Inc., IL, USA). The comparison between measurement data was analyzed by one-way ANOVA. The count data were analyzed by Pearson's $\chi^{2}$ or Fisher's exact test. The primary outcome measure was the eradication rate, the incidence of adverse events, compliance, and factors affecting eradication rates in both groups. A $p$ value of less than 0.05 was considered to be statistically significant.

\section{Results}

\subsection{Baseline data}

A total of 150 patients were enrolled in the study, all of which were analyzed by ITT. There were 76 cases of the RMMB group and 74 cases of the RMLB group. In the RMMB group, two patients lost the joint, and one patient completely stopped the drugs due to dizziness and nausea. A total of 73 patients completed the final treatment and follow-up and were included in the PP analysis. At the same time, one patient in the RMLB group lost the joint. Seventy-three patients were included in the PP analysis (Figure 1). The baseline data of age, BMI, smoking, and gastric mucosal lesions were well matched in the two groups, and there were more female patients in the RMLB group (Table 1).

\subsection{Eradication rate and influencing factors}

According to ITT analysis, the eradication rates of RMMB group and RMLB group were $80.3 \%(61 / 76)$ and $89.2 \%(66 / 74)$, respectively, and the difference was not statistically significant

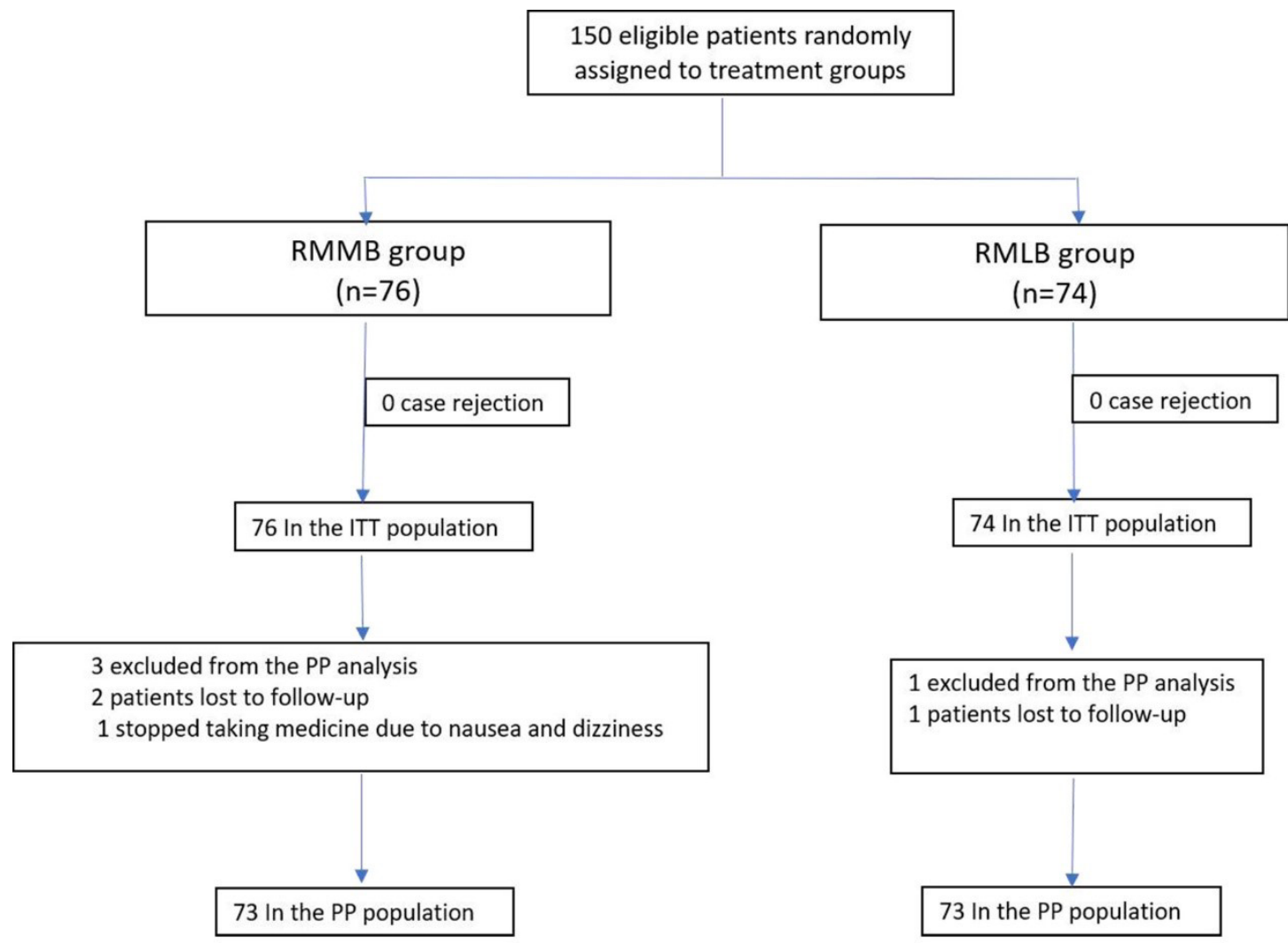

Figure 1. Flow diagram of this study. RMMB group, rabeprazole plus minocycline, metronidazole and bismuth; RMLB group, rabeprazole plus minocycline, levofloxacin and bismuth. ITT, intention-to-treat; PP, per protocol. 
$\left(\chi^{2}=2.301, p=0.129\right)$; According to the PP analysis, the eradication rates of the RMMB group and the RMLB group were $83.6 \%$ $(61 / 73)$ and $90.4 \%(66 / 73)$, respectively, and the difference was not statistically significant $\left(\chi^{2}=1.513, p=0.219\right)$. Analysis of the influence of various factors on the eradication rate found that each factor had no signficance (Table 2).

\subsection{Security and compliance}

The incidence of adverse reaction was higher in the RMMB group than in the RMLB group, but the difference was not statistically significant (Table 3). In the RMMB group, the adverse reactions were mainly due to digestive tract discomfort and dizziness, and most of them were mild and tolerable. One of the patients was severe, and the drug was discontinued and the treatment was not completed. The adverse reactions in the RMLB group were mainly dizziness and discomfort, and other digestive tract reaction was mild. No patients discontinued the drug due to adverse reactions, and the symptoms of upper abdominal pain and nausea were significantly improved compared with the RMMB group.

In this study, except for patients who were discontinued, the compliance of the remaining patients was $\geq 90 \%$, and there was no significant difference between the two groups.

\section{Discussion}

The results of this study showed that in patients with Helicobacter pylori infection allergic to penicillin, the minocycline-containing bismuth quadruple therapies achieved more than $80 \%$ eradication. Among them, the bismuth quadruple therapies with minocycline and levofloxacin achieved more than $90 \%$ eradication rate.

Table 1. The Baseline Demographic Data.

\begin{tabular}{|c|c|c|c|}
\hline Baseline data & RMMB group $(n=76)$ & RMLB group $(n=74)$ & $p$ value \\
\hline Age $(\mathrm{X} \pm \mathrm{s}$, age $)$ & $39.5 \pm 13.2$ & $38.3 \pm 11.7$ & 0.562 \\
\hline Gender (male/female) & $42 / 34$ & $25 / 49$ & 0.008 \\
\hline Smoking (example, \%) & $10(13.2)$ & $8(10.8)$ & 0.872 \\
\hline Human body mass index $\left(\mathrm{kg} / \mathrm{m}^{2}\right)$ & $22.9 \pm 3.3$ & $23.0 \pm 3.8$ & 0.885 \\
\hline Gastroscopic findings (peptic ulcer/non-peptic ulcer, \%) & $7 / 29(19.4)$ & $3 / 16(15.8)$ & 0.667 \\
\hline
\end{tabular}

Table 2. Comparisons of Eradication Rates of The Two Groups.

\begin{tabular}{|c|c|c|c|c|}
\hline \multirow{2}{*}{ project } & \multicolumn{2}{|c|}{ RMMB group $(n=76)$} & \multicolumn{2}{|c|}{ RMLB group $(n=74)$} \\
\hline & Eradication rate (\%) & $p$ value & Eradication rate $(\%)$ & $p$ value \\
\hline Age (years) & & 0.326 & & 0.990 \\
\hline$<45$ & $80.4(41 / 5)$ & & $90.4(47 / 52)$ & \\
\hline$\geq 45$ & $90.9(20 / 22)$ & & $90.5(19 / 21)$ & \\
\hline Gender & & 0.788 & & 0.677 \\
\hline Male & $82.5(33 / 40)$ & & $87.5(21 / 24)$ & \\
\hline Female & $84.8(28 / 33)$ & & $91.8(45 / 49)$ & \\
\hline Human body mass index $\left(\mathrm{kg} / \mathrm{m}^{2}\right)$ & & 0.965 & & 0.075 \\
\hline$<24$ & $83.7(36 / 43)$ & & $96.1(49 / 51)$ & \\
\hline$\geq 24$ & $83.3(25 / 3)$ & & $77.3(17 / 22)$ & \\
\hline Endoscopic performance & & 0.023 & & 0.414 \\
\hline Peptic ulcer & $-(3 / 7)$ & & $-(3 / 3)$ & \\
\hline Non-peptic ulcer & $88.5(23 / 26)$ & & $81.3(13 / 16)$ & \\
\hline
\end{tabular}

Table 3. Side effects of different treatment regimens.

\begin{tabular}{|c|c|c|c|}
\hline & $\operatorname{RMMB}(n=76)$ & $\operatorname{RMLB}(n=74)$ & $p$ values \\
\hline Side effects of treatment, n (\%) & $36(47.4)$ & $25(33.8)$ & 0.189 \\
\hline Nausea & $12(15.8)$ & $3(4.1)$ & 0.017 \\
\hline Upper abdominal discomfort & $25(32.9)$ & $9(12.2)$ & 0.002 \\
\hline Dizziness & $18(23.7)$ & $15(20.3)$ & 0.614 \\
\hline Insomnia & $3(3.9)$ & $3(4.1)$ & 1.000 \\
\hline Diarrhea & $8(10.5)$ & $4(5.4)$ & 0.248 \\
\hline Abnormal taste & $6(7.9)$ & $8(10.8)$ & 0.539 \\
\hline Arthralgia & 1 & 0 & \\
\hline Rash & 0 & 1 & \\
\hline Oral ulcer & 0 & 1 & \\
\hline
\end{tabular}

RMMB group, rabeprazole plus minocycline, metronidazole and bismuth; RMLB group, rabeprazole plus minocycline, levofloxacin and bismuth. 
According to Graham's standard, it is a better eradication effect (Graham et al., 2007). And no noticeable adverse reactions appear, it is worth promoting the use of patients with penicillin allergy.

At present, the global $H p$ infection rate is very high, especially in countries and regions where economy is underdeveloped. China is a large country with Helicobacter pylori infection, and the $H p$ infection rate is more than 50\% (Zhang et al., 2014). Due to the close correlation between $H p$ infection and diseases such as peptic ulcer, gastric cancer and MALT lymphoma, many domestic and foreign guidelines recommend active eradication therapy for $\mathrm{H} p$-infected patients. However, in recent years, the resistance of $H p$ has become more and more higher, which has made it more and more challenging to eradicate. A recent study in Beijing showed that (Zhang et al., 2015b) Hp resistance rates to amoxicillin, clarithromycin, metronidazole, levofloxacin and tetracycline were $4.4 \%, 52.6 \%, 54.8 \%, 63.4 \%$ and $7.3 \%$, respectively. For patients who are allergic to penicillin, under the premise of not being able to use amoxicillin, a tetracyclinebased bismuth quadruple protocol is recommended, including tetracycline combined with nitroimidazole, fluoroquinolone or furazolidone. However, in Beijing, due to the high drug resistance of clarithromycin and metronidazole, and the large side effects of tetracycline and furazolidone, the clinical application of this quadruple protocol is limited. This study explored the use of semi-synthetic tetracycline, minocycline instead of tetracycline to form an bismuth quadruple therapy, and observed its effect in patients with penicillin allergy, the results are ideal.

As a semi-synthetic tetracycline widely used in clinical practice, minocycline has a strong drug effect, fewer side effects and convenient use. Previous in vitro susceptibility studies have found that $H p$ has a low resistance to minocycline, similar to tetracycline (Bai et al., 2015; Horiki et al., 2009). There are also many cases of minocycline-based triple-linked regimen based on drug sensitivity for successful eradication of $H p$ infection in the clinic (Nakajima et al., 2012; Konno et al., 2010; Asaumi et al., 2003). At present, the clinical research on the application of minocycline in $H p$ infection is mostly aimed at re-treatment patients. Our previous study showed that in the $H p$ retreatment patients, the bismuth quadruple therapies with minocycline/amoxicillin was used, and the eradication rate was 79.4\% (ITT analysis) and $84.1 \%$ (PP analysis) (Zhang et al., 2015a, 2017). The efficacy of this program is ideal. But there are also different research results reported. A study in Italy included 45 patients with $H p$ infection who underwent thirdline therapy (Ierardi et al., 2014) and were randomized into two groups: rabeprazole/riflbutin/minocycline/bismuth group and rabeprazole/tinidazole/minocycline/bismuth group. The course of treatment is 10 days. The eradication rates of the two groups were $77.7 \%, 51.9 \%$ in ITT analysis and $84.0 \%$ and $51.9 \%$ in PP analysis, suggesting that the eradication effect of the bismuth quadruple therapies with minocycline and tinidazole was not satisfactory. However, the subjects were all highly resistant retreatment patients, and the treatment was only 10 days, which may affect their eradication rate.

In this study, the eradication rate of the RMMB regimen containing minocycline and metronidazole was $80.3 \%$ (ITT analysis) and $83.6 \%$ (PP analysis). The results show that the eradication effect is not ideal, and the reason may be due to the high drug resistance of metronidazole. The dosage of metronidazole is ( $0.4 \mathrm{~g}$, TID), and studies have shown that increasing the dose of metronidazole can overcome the drug resistance to some extent (Malfertheiner et al., 2017; Fallone et al., 2016). However, the adverse reactions in the clinical application of metronidazole are more obvious with larger doses, the most important of which is digestive tract discomfort, including nausea. It was also observed in this study that the gastrointestinal response of the metronidazole group was significantly higher than that of the levofloxacin group. Another adverse reaction of metronidazole is abnormal nervous system, dizziness, headache and visual impairment. After increasing the dose, there may even be convulsions and neuroinflammation (Chen, 2018). And minocycline also causes dizziness through vestibular nerve reflex, and combined with high doses of metronidazole may aggravate the adverse reactions of the nervous system. Therefore, whether the combination of metronidazole and minocycline can be considered as a modified standard bismuth quadruple therapies should be carefully considered.

In this study, the RMLB regimen containing minocycline and levofloxacin achieved better eradication effects, $89.2 \%$ (ITT analysis) and $90.4 \%$ (PP analysis). Levofloxacin is a commonly used drug for the eradication of Helicobacter pylori and is recommended for the remedial treatment of Helicobacter pylori in domestic and international consensus. And the research confirmed that the eradication effect is ideal (Malfertheiner et al., 2017). Since the abuse of fluoroquinolones in China has led to the high primary resistance of $H p$ to its clinical application, it is recommended in recent national consensus opinion in China that fluoroquinolones are not used in routine first-line treatment. But it can be used in patients with second-line or penicillin allergy (The Chinese Society of Digestive Diseases, 2017). In recent years, studies have shown that the combination of levofloxacin and amoxicillin in the quadruple regimen has a better eradication rate and fewer side effects (Ahn et al., 2017). For patients with allergic to penicillin, it is routinely recommended to replace amoxicillin with tetracycline, and its eradication effect with levofloxacin has been confirmed (Hsu et al., 2014). In this paper, minocycline is used to replace the tetracycline which is difficult to obtain in clinical practice, and the bismuth quadruple therapies composed of levofloxacin has achieved an ideal eradication effect.

In terms of adverse reactions, there was no significant difference in the overall incidence between the two groups of patients. The symptoms of dizziness were evident in both groups, mainly related to the reversible vestibular nerve reaction of minocycline, which was characterized by vertigo, tinnitus, ataxia, nausea, vomiting and other vestibular dysfunction. Symptoms often occur when the drug is initially administered and recovers after 24 to 48 hours of withdrawal. Patients in the levofloxacincontaining regimen had a lower gastrointestinal response than the metronidazole group, and patients were better tolerated. This program can be recommended as a better choice for the eradication of patients with penicillin allergy.

This study showed that in patients with penicillin allergy, the replacement effect of amoxicillin with minocycline was an ideal regimen for eradication. And the bismuth quadruple therapies 
composed of minocycline and levofloxacin has a better eradication rate and fewer side effects, which is more ideal. However, there is no drug sensitivity test in this study. It is difficult to determine whether the patients in the two groups have similar antibiotic resistance. Moreover, this is a single-center study, and the patients may have deviations. Therefore, the conclusion of this study needs to be further studied.

\section{Ethical approval}

Ethical approval for this study was provided by the Ethical Committee of Beijing Jishuitan Hospital. This study was performed in accordance with the ethical principles of the Declaration of Helsinki (Association 2009).

\section{Conflict of interest}

No conflicts of interest were declared by the authors.

\section{Funding}

This study was supported by Beijng Jishuitan Hospital Nova Program (NO: XKXX201609).

\section{Acknowledgements}

This research did not receive any specific grant from funding agencies in the public, commercial, or not-for-profit sectors.

\section{References}

Ahn, H. J., Kim, D. P., Chu, M. S., Yun, H. J., Kim, S. H., Lee, S. W., \& Lee, D. S. (2017). Efficacy and safety of the triple therapy containing ilaprazole, levofloxacin, and amoxicillin as first-line treatment in helicobacter pylori infections. Gastroenterology Research and Practice, 2017, 1654907. http://dx.doi.org/10.1155/2017/1654907. PMid:28539935.

Asaumi, N., Masuda, K., Yoshida, C., Kiguchi, T., Niiya, M., Niiya, K., \& Tanimoto, M. (2003). Helicobacter pylori eradication based upon a drug sensitivity test effectively increased platelet count in a patient with refractory idiopathic thrombocytopenic purpura. The Japanese Journal of Clinical Hematology, 44(7), 480-482. PMid:12931570.

Bai, P., Zhou, L. Y., Xiao, X. M., Luo, Y., \& Ding, Y. (2015). Susceptibility of Helicobacter pylori to antibiotics in Chinese patients. Journal of Digestive Diseases, 16(8), 464-470. http://dx.doi.org/10.1111/17512980.12271. PMid:26147515.

Chen, X. (2018). Clinical safety medication study of metronidazole. Clinical Medicine, 8, 53. http://dx.doi.org/10.3969/j.issn.10066845.2018.08.046.

Fallone, C. A., Chiba, N., van Zanten, S. V., Fischbach, L., Gisbert, J. P., Hunt, R. H., Jones, N. L., Render, C., Leontiadis, G. I., Moayyedi, P., \& Marshall, J. K. (2016). The Toronto consensus for the treatment of helicobacter pylori infection in adults. Gastroenterology, 151(1), 5169. http://dx.doi.org/10.1053/j.gastro.2016.04.006. PMid:27102658.

Graham, D. Y., Lu, H., \& Yamaoka, Y. (2007). A report card to grade helicobacter pylori therapy. Helicobacter, 12(4), 275-278. http:// dx.doi.org/10.1111/j.1523-5378.2007.00518.x. PMid:17669098.

Horiki, N., Omata, F., Uemura, M., Suzuki, S., Ishii, N., Iizuka, Y., Fukuda, K., Fujita, Y., Katsurahara, M., Ito, T., Cesar, G. E., Imoto, I., \& Takei, Y. (2009). Annual change of primary resistance to clarithromycin among Helicobacter pylori isolates from 1996 through 2008 in
Japan. Helicobacter, 14(5), 86-90. http://dx.doi.org/10.1111/j.15235378.2009.00714.x. PMid:19751432.

Hsu, P. I., Chen, W. C., Tsay, F. W., Shih, C. A., Kao, S. S., Wang, H. M., Yu, H. C., Lai, K. H., Tseng, H. H., Peng, N. J., Chen, A., Kuo, C. H., \& Wu, D. C. (2014). Ten-day quadruple therapy comprising proton-pump inhibitor, bismuth, tetracycline,and levofloxacin achieves a high eradication rate for Helicobactor pylori infection after failure of sequential therapy. Helicobacter, 19(1), 74-79. http:// dx.doi.org/10.1111/hel.12085. PMid:24033865.

Ierardi, E., Giangaspero, A., Losurdo, G., Giorgio, F., Amoruso, A., De Francesco, V., Di Leo, A., \& Principi, M. (2014). Quadruple rescue therapy after first and second line failure for Helicobacter pylori treatment: comparison between two tetracycline-based ragimens. Journal of Gastrointestinal and Liver Diseases; JGLD, 23(4), 367-370. http://dx.doi.org/10.15403/jgld.2014.1121.234.qrth. PMid:25531993.

Konno, T., Motoori, S., Iwamoto, N., Miyazawa, T., Saito, S., Kitagawa, N., Saisho, H., Furuse, J., \& Itabashi, M. (2010). A case of mucosaassociated lymphoid tissue lymphoma with penicillin allergy successfully treated with levofloxacin, minomycin and rabeprazole. Cancer \& Chemotherapy, 37(10), 1961-1962. PMid:20948264.

Lesaffre, E., \& de Klerk, E. (2000). Estimating the power of complianceimproving methods. Controlled Clinical Trials, 21(6), 540-551. http://dx.doi.org/10.1016/S0197-2456(00)00088-X. PMid:11146148.

Malfertheiner, P., Megraud, F., O’morain, C. A., Gisbert, J. P., Kuipers, E. J., Axon, A. T., Bazzoli, F., Gasbarrini, A., Atherton, J., Graham, D. Y., Hunt, R., Moayyedi, P., Rokkas, T., Rugge, M., Selgrad, M., Suerbaum, S., Sugano, K., \& El-Omar, E. M. (2017). European Helicobacter and Microbiota Study Group and Consensus panel. Management of Helicobacter pylori infection - the Maastricht V/Florence Consensus Report. Gut, 66(1), 6-30. http://dx.doi.org/10.1136/ gutjnl-2016-312288. PMid:27707777.

Nakajima, S., Inoue, H., Inoue, T., \& Maruoka, Y. (2012). Multipleantibiotic-resistant Helicobacter pylori infection eradicated with a tailor-made quadruple therapy. Journal of Gastroenterology and Hepatology, 27(3, Suppl. 3), 108-111. http://dx.doi.org/10.1111/j.14401746.2012.07069.x. PMid:22486882.

The Chinese Society of Digestive Diseases. (2017). The Fifth Chinese national consensus report on management of helicobacter pylori infection. Chinese Journal of Digestion, 37(6), 364-378. http://dx.doi. org/10.3969/j.issn.1008-7125.2017.06.006.

Zhang, L., Zhou, L., Song, Z., Ding, Y., \& Bai, P. (2015a). The application of minocycline quadruple protocol and individualized regimen in the retreatment of Helicobacter pylori infection. Chinese Journal of Integrative Medicine, 54(12), 1013-1017. http://dx.doi.org/10.3760/ cma.j.issn.0578-1426.2015.12.005.

Zhang, Ln., Lan, Y., Fan, Y., Zhang, Y., \& Wang, Q. (2017). The application of minocycline-based quadruple protocol in the retreatment of Helicobacter pylori infection. Chinese General Practice, 20(18), 2237-2240. http://dx.doi.org/10.3969/j.issn.1007-9572.2017.18.014.

Zhang, M., Zhou, Y. Z., Li, X. Y., Tang, Z., Zhu, H. M., Yang, Y., \& Chhetri, J. K. (2014). Seroepidemiology of Helicobacter pylori infection in elderly people in the Beijing region, China. World Journal of Gastroenterology, 20(13), 3635-3639. http://dx.doi.org/10.3748/ wjg.v20.i13.3635. PMid:24707148.

Zhang, Y. X., Zhou, L. Y., Song, Z. Q., Zhang, J. Z., He, L. H., \& Ding, Y. (2015b). Primary antibiotic resistance of Helicobacter pylori strains isolated from patients with dyspeptic symptoms in Beijing: a prospective serial study. World Journal of Gastroenterology, 21(9), 2786-2792. http://dx.doi.org/10.3748/wjg.v21.i9.2786. PMid:25759550. 